\title{
Efficacy of EUS-RFA in pancreatic tumors: Is it ready for prime time? A systematic review and meta-analysis
}

\section{(ㄷ)(요 $\odot$}

\section{Authors}

Amaninder Dhaliwal ${ }^{1}$, Sindhura Kolli ${ }^{2}$, Banreet Singhg Dhindsa ${ }^{3}$, Jacqueline Choa ${ }^{3}$, Harmeet Singh Mashiana ${ }^{1}$, Daryl Ramai $^{4}$, Saurabh Chandan ${ }^{1}$, Neil Bhogal ${ }^{1}$, Harlan Sayles ${ }^{5}$, Ishfaq Bhat ${ }^{1}$, Shailender Singh ${ }^{1}$, Douglas G. Adler ${ }^{6}$

Institutions

1 Division of Gastroenterology and Hepatology, University of Nebraska Medical Center, Omaha, Nebraska, United States

2 Department of Bariatrics, NYU Langone Medical Center, New York, New York, United States

3 Department of Internal Medicine, University of Nevada Las Vegas School of Medicine, Las Vegas, Nevada, United States

4 Department of Internal Medicine, The Brooklyn Hospital Center, Brooklyn, New York, United States

5 Department of Biostatistics, University of Nebraska Medical Center, Omaha, Nebraska, United States

6 University of Utah School of Medicine, Gastroenterology and Hepatology, Salt Lake City, Utah, United States

submitted 16.4 .2020

accepted after revision 12.6.2020

Bibliography

Endoscopy International Open 2020; 08: E1243-E1251

DOI 10.1055/a-1221-5012

ISSN 2364-3722

(c) 2020. The Author(s).

This is an open access article published by Thieme under the terms of the Creative Commons Attribution-NonDerivative-NonCommercial License, permitting copying and reproduction so long as the original work is given appropriate credit. Content may not be used for commecial purposes, or adapted, remixed, transformed or built upon. (https://creativecommons.org/licenses/by-nc-nd/4.0/)

Corresponding author

Douglas G. Adler, MD, FACG, AGAF, FASGE, Professor of Medicine, Director of Therapeutic endoscopy, Director, GI fellowship program, Gastroenterology and Hepatology, University of Utah School of Medicine, Huntsman Cancer Center, 30 N 1900 E, Room 4R118, Salt Lake City, Utah 84132, United States

Fax: +1-801-581-8007

douglas.adler@hsc.utah.edu

\section{ABSTRACT}

Background and study aims Endoscopic ultrasoundguided radiofrequency ablation (EUS-RFA) can be used in patients with unresectable pancreatic ductal adenocarcinoma (PDAC). We performed a systematic review and metaanalysis to evaluate the efficacy of EUS-RFA in treatment of locally advanced unresectable PDAC and other pancreatic tumors.

Patients and methods A comprehensive search was done of multiple electronic databases and conference proceedings including PubMed, EMBASE, Web of Science databases, Google Scholar and manual search of references (from inception through May 2019) to identify the studies reporting use of EUS-RFA for pancreatic lesions. The primary outcome was to evaluate technical and clinical success of the procedure. The secondary outcome was to study overall adverse events (AEs).

Results Thirteen studies reporting 165 EUS-RFA procedures on 134 patients were included. Of 134 patients, $27.94 \%$ (38) had unresectable locally advanced PDAC, $40 \%$ (53) had PNETs, $3 \%$ (4) had metastasis to the pancreas and $30 \%(41)$ had other lesions. The pooled technical success rate calculated out of the total number of procedures was $100 \%(95 \% \mathrm{Cl}[99.18-100], \mathrm{I} 2=0 \%)$. The pooled clinical success rate calculated out of the total number of patients was $91.58 \%$ (95\% Cl [82.5-98.08], I2=21.5\%). The pooled overall $\mathrm{AE}$ rates were $14.67 \%(95 \% \mathrm{Cl}$ [4.77-27.46], $\mathrm{I} 2=$ $56.19 \%$ ) out of which abdominal pain was the most common with $9.82 \%(95 \% \mathrm{Cl}[3.34-18.24], 12=23.76 \%)$. Low to moderate heterogeneity was noted.

Conclusion EUS-RFA has high technical (100\%) and clinical success $(91.5 \%)$ rates. Further multicenter trials are needed to further validate our findings.

\section{Introduction}

Endoscopic ultrasound-guided radiofrequency ablation (EUSRFA) works by placing an RFA probe, inserted through an EUS needle, under EUS color Doppler guidance, into a target pancreatic lesion, delivering various energy outputs directly related to the size of the lesion for a variable amount of time or until an 
electrical impedance of $>500$ Ohms is detected, suggesting that coagulative necrosis has occurred within the lesion [1]. This is confirmed by postoperative imaging with a computed tomography (CT) scan.

Unfortunately, given current National Comprehensive Cancer Network (CCN) guidelines, approximately $80 \%$ of patients with pancreatic ductal adenocarcinoma (PDAC) will have disease deemed unresectable at the time of diagnosis. [2] This creates a potential opportunity for EUS-RFA to provide a palliative option in their treatment plan. In cases of unresectable or locally advanced PDAC, EUS-RFA is a developing option with the potential to reduce or downstage the tumor burden. EUS-RFA is potentially a valuable tool for reducing tumor size by inducting coagulative necrosis from direct thermal destruction and/or triggering immunostimulation and antitumor antigens $[3,4]$.

Use of EUS-RFA in the pancreas also extends to treatment and ablation of small pancreatic neuroendocrine tumors (PNETs), pancreatic cystic neoplasms (PCN) which include mucinous pancreatic cystic neoplasms (MCN), and intraductal papillary mucinous neoplasms (IPMN) and other pancreatic tumors measuring $<3 \mathrm{~cm} \mathrm{[1]}$.

To date, several studies have evaluated use of EUS-RFA in the pancreas. The primary end point of this systematic review and meta-analysis is to evaluate technical and clinical success of EUS-RFA in locally advanced PDAC and other pancreatic tumors. Secondary endpoints were to recognize and study both early and late adverse events (AEs), given the thermosensitivity of pancreatic tissue.

\section{Methods}

\section{Search strategy}

We conducted a comprehensive search of several databases and conference proceedings including PubMed, EMBASE, MEDLINE, Google-Scholar, Cochrane, LILACS, SCOPUS, and Web of Science databases (earliest inception to May 2019). We followed the preferred reporting items for systematic reviews and meta-Analyses (PRISMA) guidelines to identify studies reporting on EUS RFA in pancreatic tumors [5]. An experienced medical librarian, using inputs from the study authors, helped with the literature search.

Key words used in the literature search included a combination of 'EUS, 'Endoscopic, and 'Ultrasound', 'radiofrequency, ablation', 'tumor and pancreatic. The search was restricted to studies in human subjects and published in English language in peer-reviewed journals. Two authors (BD, JC) independently reviewed the titles and abstracts of studies identified in the primary search and excluded studies that did not address the research question, based on prespecified exclusion and inclusion criteria. The full text of remaining articles was reviewed to determine whether they contained relevant information. Any discrepancies in article selection were resolved by consensus, and in discussion between the co-authors.

The bibliographic section of the selected articles, as well as the systematic and narrative articles on the topic were manually searched for additional relevant articles.

\section{Study selection}

We included studies that evaluated the efficacy of and AEs associated with EUS-RFA in pancreatic tumors. Studies were included as long as they provided data needed for the analysis. Exclusion criteria were as follows: (1) studies using EUS-RFA in tumors other than pancreas, (2) studies performed in the pediatric population (Age <18 years), and (3) studies not published in the English language.

In cases of multiple publications from the same cohort and/ or overlapping cohorts, data from the most recent and/or most appropriate comprehensive report were included.

\section{Data abstraction and quality assessment}

Data on study-related outcomes in the individual studies were abstracted onto a standardized form by at least three authors $(B D, A D, J C)$, and two authors (BD, $A D)$ performed the quality scoring independently.

The data collection was performed as number of reported events $(n)$ out of the total number of patients $(N)$ from each study. The collected data was treated akin to single group cohort studies and therefore we used the Newcastle-Ottawa scale for cohort and case-control studies to assess the quality of studies [6]. This quality score consisted of 8 questions, the details of which are provided in $>$ Table 1 .

\section{EUS-RFA technique}

EUS-RFA is a procedure that requires a unipolar probe deployed under ultrasound guidance to the center of the tumor. A generator is activated to release a certain wattage for a set amount of time that varies and specific to each RFA model. The probe is

Table 1 Quality assessment of the study with Newcastle Ottawa Scale.

\begin{tabular}{|l|l|l|l|l|}
\hline Author & Year & \multicolumn{3}{|c|}{ Newcastle-Ottawa Scale } \\
\hline & & $\begin{array}{l}\text { Selec- } \\
\text { tion }\end{array}$ & $\begin{array}{l}\text { Compar- } \\
\text { ability }\end{array}$ & $\begin{array}{l}\text { Out- } \\
\text { come }\end{array}$ \\
\hline Oleinikov [11] & 2019 & $* * *$ & $*$ & $* * *$ \\
\hline Barthet [1] & 2018 & $* * *$ & $*$ & $* *$ \\
\hline Scopelliti [3] & 2018 & $* * *$ & $*$ & $* *$ \\
\hline Crino [9] & 2018 & $* * *$ & $*$ & $* * *$ \\
\hline De la Serna [8] & 2018 & $* *$ & $*$ & $*$ \\
\hline Choi [7] & 2018 & $* * *$ & $*$ & $* * *$ \\
\hline Thosani [12] & 2018 & $* *$ & $*$ & $* *$ \\
\hline Goyal [13] & 2017 & $* *$ & $*$ & $*$ \\
\hline Malikowski [14] & 2017 & $* *$ & $*$ & $*$ \\
\hline Wang [16] & 2016 & $* * *$ & $*$ & $*$ \\
\hline Song [4] & 2016 & $* * *$ & $*$ & $*$ \\
\hline Lakhtakia [10] & 2016 & $* * *$ & $*$ & $* * *$ \\
\hline Pai [15] & 2015 & $* *$ & $*$ & $* *$ \\
\hline
\end{tabular}


then rotated, and the process is repeated from one to four times. An internal cooling system is sequentially employed to prevent thermogenic damage to surrounding tissue. Postoperative computed tomography (CT) scans were performed to compare the change in size of the enhanced tissue of the tumor to a preoperative CT, as well as to assess for any complications [3].

\section{EUS-RFA needles and catheters}

Two types of RFA probes were used in our meta-analysis. A STARmed needle of different sizes (18G/19G/22 G) was most commonly used to deliver RFA energy [3, 4, 7-12]. This operative needle is associated with a pump cooling the needle with help of chilled saline which prevents charring of the tip and improves accuracy of ablation.

The rest of the studies used the Habib catheter for delivery of RFA energy [13-16]. This is a monopolar electrode without a cooling system which is inserted inside a standard EUS FNA needle and attached to an electrosurgical generator [17]. The different RFA needles/catheters and ablative energy settings used in our study population are listed in $\mathbf{r}$ Table 2.

\section{Outcomes assessed}

Primary outcome

Technical success and clinical success of EUS-RFA in pancreatic tumors

- Table2 EUS-RFA Needle/catheter with RFA energy used in different studies.

\begin{tabular}{|l|l|l|}
\hline Author & Catheter/Needle used & RFA Energy \\
\hline Oleinikov [11] & STARmed 19G & $50 \mathrm{~W}$ \\
\hline Barthet [1] & STARmed 18G & $50 \mathrm{~W}$ \\
\hline Scopelliti [3] & STARmed 18G & $\begin{array}{l}\text { lesions }>3 \mathrm{~cm}- \\
30 \mathrm{~W} \text { and Lesions } \\
<3 \mathrm{~cm}-20 \mathrm{~W}\end{array}$ \\
\hline Crino [9] & STARmed 18G & $30 \mathrm{~W}$ \\
\hline De la Serna [8] & STARmed 18G and 19G & $50 \mathrm{~W}$ \\
\hline Choi [7] & STARmed 18G and 19G & $50 \mathrm{~W}$ \\
\hline Thosani [12] & STARmed 19G and 22 G & - \\
\hline Goyal [13] & Habib catheter (1Fr)/22 G & $10 \mathrm{~W}$ \\
\hline Malikowski [14] & Habib catheter & $10 \mathrm{~W}$ \\
\hline Wang [16] & Habib catheter/22 G & $10 \mathrm{~W}-15 \mathrm{~W}$ \\
\hline Song [4] & STARmed 18G & $20 \mathrm{~W}-50 \mathrm{~W}$ \\
\hline Lakhtakia [10] & STARmed 19G & $50 \mathrm{~W}$ \\
\hline Pai [15] & $\begin{array}{l}\text { Habib catheter (1Fr)/19 } \\
\text { and 22 G }\end{array}$ & $5-25 \mathrm{~W}$ \\
\hline EUS-RFA, endoscopic ultrasound radiofrequency ablation. \\
\hline
\end{tabular}

\section{Total AEs \\ 2. Analysis of individual AEs}

\section{Definitions}

Technical success was defined as the successful placement of the needle within the pancreatic lesions with safe margins from the surrounding vital structures to avoid potential thermal injuries and application of radiofrequency ablation based on impedance.

Clinical success was defined as decrease in lesion size and presence of hypodense area (necrosis) on CT scan after the procedure in case of unresectable locally advanced pancreatic adenocarcinoma, metastatic pancreatic lesions, and other benign pancreatic tumors. In case of PNETs, it was defined as improvement in symptoms along with decrease in lesion size and presence of hypodense area on CT scan.

Overall, locally advanced unresectable pancreatic carcinoma was defined in two studies as per 2016 NCCN guidelines: in cases of tumor contact greater than $180^{\circ}$ with major arteries such as the celiac trunk (CT), the superior mesenteric artery (SMA) and the first jejunal branch, involvement of the aorta, unreconstructable involvement of vessels such as the hepatic artery (HA), the superior mesenteric vein (SMV), and the portal vein (PV), and contact with the most proximal draining jejunal branch into SMV and SMV or PV occlusion (due to the tumor or bland thrombus).

AEs were divided into early ( $<7$ days) and late ( $>7$ days to 3 months). In the early group, subgroup analysis was then done to calculate the pooled rates of individual AEs.

\section{Statistical analysis}

We used meta-analysis techniques to calculate the pooled estimates for each outcome following the methods suggested by DerSimonian and Laird using the random-effects model [18]. When incidence of an outcome was zero in a study, a continuity correction of 0.5 was added to the number of incident cases before the study was entered into the statistical model [19]. We assessed heterogeneity between study-specific estimates by using Cochran $\mathrm{Q}$ statistical test for heterogeneity, and the $\mathrm{I}^{2}$ statistics. [20,21] In this, values $<30 \%, 30 \%$ to $60 \%, 61 \%$ to $75 \%$, and $>75 \%$ were suggestive of low, moderate, substantial, and considerable heterogeneity, respectively [22].

All analyses were performed using Comprehensive Meta-Analysis (CMA) software, version 3 (BioStat, Englewood, New Jersey, United States).

\section{Results}

\section{Search results and population characteristics}

From an initial pool of 84 studies, 13 studies reported use of EUS-RFA in pancreatic lesions [1,3,4,7-16]. The schematic diagram of study selection as per PRISMA guidelines is illustrated in $>$ Fig. 1.

Mean age was 61.42 (range 45-69) years with a predominantly male population. The "mean" median lesion was $27.21 \mathrm{~mm}$ (range 9-49.2). Mean follow-up post-procedure 




- Fig. 1 Study selection process in accordance with preferred reporting items for systematic reviews and meta-analysis statement.

was 6.5 months (range 1 -12). Four studies used Habib catheters and nine studies used STARmed needles. Patient demographics are described in $>$ Table 3.

\section{Characteristics and quality of included studies}

Twelve studies were prospective and one study was retrospective. Three studies were multicenter and the rest were single center. No studies were population based. All studies reported adequately on the technical success, adverse events, assessment, and factors were comparable between the study groups. Ten of 13 studies reported on location of pancreatic tumor and clinical success. Overall, five studies were considered of high quality, six were of medium quality and two were low-quality studies. A detailed assessment of study quality is given in $\triangleright \mathbf{T a}$ ble 1.

\section{Meta-analysis outcomes}

A total of 134 patients were included in the analysis and 165 EUS-RFA procedures were performed on these patients. Of 134 patients, $27.94 \%$ (38) had unresectable locally advanced PDAC, $40 \%$ (53) had PNETs, $3 \%$ (4) had metastasis to the pancreas and $30 \%$ (41) had other lesions which included IPMNs, mucinous cysts, solid pseudopapillary neoplasm, and microcystic adenomas. The most common lesion location was the head (51.6\%) followed by the body of the pancreas (39.5\%).

The primary outcomes of the study were technical and clinical success of EUS-RFA. The pooled technical success rate cal- culated out of the total number of procedures was 100\% (95\% $\left.\mathrm{Cl}[99.18-100], \mathrm{I}^{2}=0 \%\right)$. The pooled clinical success rate calculated out of the total number of patients was $91.58 \%(95 \% \mathrm{Cl}$ [82.5-98.08], $I^{2}=21.5 \%$ ( Fig. 2, $>$ Fig. 3 ).

The secondary outcome was to assess AEs associated with EUS-RFA. The pooled overall AEs were 14.67\% (95\% CI [4.7727.46], $\mathrm{I}^{2}=56.19 \%$ ) ( $>$ Fig.4). In individual AE analysis, abdominal pain was the most common side effect $9.82 \%(95 \% \mathrm{Cl}$ [3.34-18.24], $\left.\mathrm{I}^{2}=23.76 \%\right)$. No bleeding was noted, and postprocedure pancreatitis was noted in two patients. Perforation and procedure-related infections were noted in one patient each ( $>$ Table 4$)$.

Delayed AEs (> 7 days) were reported in two studies including three patients $[1,11]$. In the Oleinokov study, two patients developed mild pancreatitis between 7 and 10 days post-procedure, which resolved on the second or third day of the hospitalization. In the Barthet study, one patient developed stenosis of the main pancreatic duct 1 week after the procedure and underwent pancreatic duct stenting.

\section{Validation of meta-analysis results}

Sensitivity analysis

To assess whether any one study had a dominant effect on the meta-analysis, we excluded one study at a time and analyzed its effect on the main summary estimate. On this analysis, no single study significantly affected the outcome or the heterogeneity.

\section{Heterogeneity}

We assessed dispersion of the calculated rates using $I^{2}$ percentage values. The $I^{2}$ tells us what proportion of the dispersion is true versus chance [19]. The pooled rates of technical success and clinical success showed low heterogeneity and pooled overall early AE rates showed moderate heterogeneity.

\section{Publication bias}

Publication bias was difficult to estimate properly as we were evaluating one-arm studies with dichotomous outcomes and most of the studies were fairly small.

\section{Discussion}

The primary measured outcomes of the studies included in this meta-analysis were the technical and clinical success of EUSRFA in treating pancreatic lesions, both malignant and potentially malignant. The pooled technical success rate was $100 \%$ $\left(95 \% \mathrm{Cl}[99.18-100], \mathrm{I}^{2}=0 \%\right)$ for all types of pancreatic lesions. In the 13 studies we examined, 12 studies reached $100 \%$ technical success, including Barthet et al who examined the highest number of lesions at 31 . The remaining one study had technical success of $94 \%$. Scopelliti et al, who had a $100 \%$ technical success rate, pointed out logistical and anatomical limitations such as scarring from previous radiation or inability to maneuver the stiff probe to the target site [3].

The pooled clinical success rate was $91.58 \%$ (95\% Cl [82.5$98.08], I^{2}=21.5 \%$ ). Given the wide range of pancreatic lesions being treated, the success rate was defined as decrease in tu- 


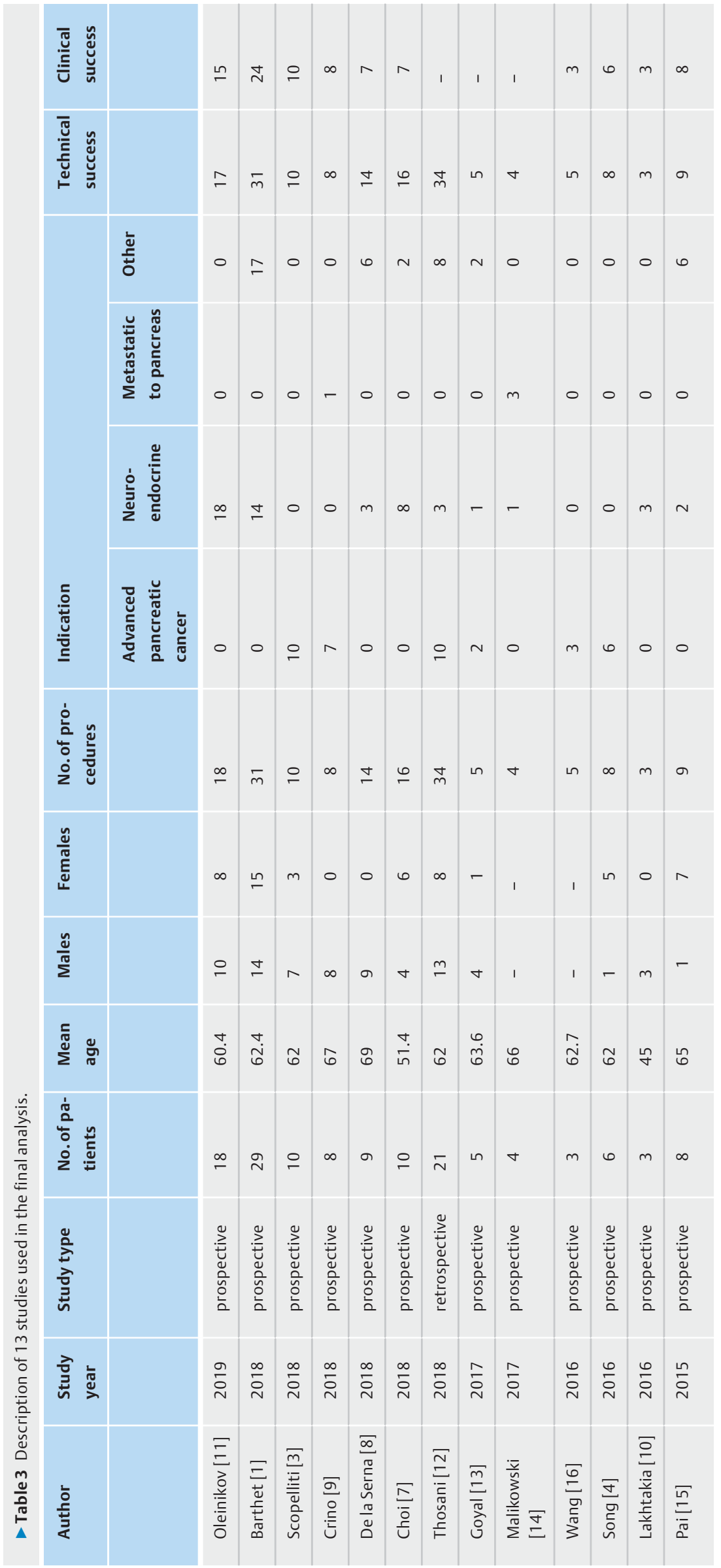


Study

$(95 \% \mathrm{Cl})$

$\begin{array}{ccc}\% & \text { Technical } & \text { Number of } \\ \text { weight } & \begin{array}{c}\text { success } \\ \text { (out of no. of } \\ \text { procedures) }\end{array}\end{array}$

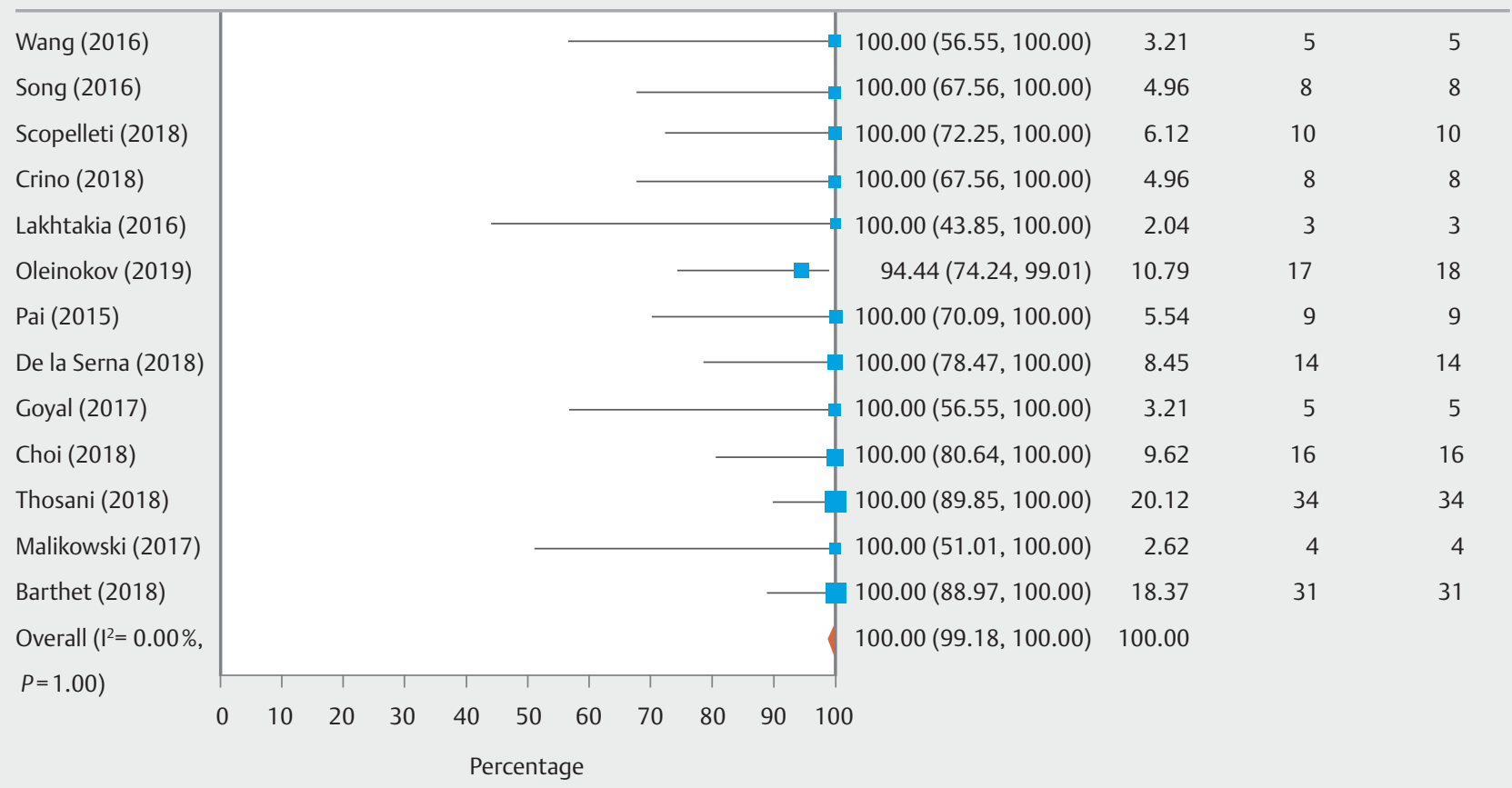

- Fig. 2 Overall pooled technical success rate of EUS-RFA in pancreatic tumors.



- Fig. 3 Overall pooled clinical success rate of EUS-RFA in pancreatic tumors. 
Study

$\begin{array}{cccc}\text { ES } & \begin{array}{c}\% \\ (95 \% \mathrm{Cl})\end{array} & \begin{array}{c}\text { Total } \\ \text { adverse } \\ \text { events }\end{array} & \begin{array}{c}\text { Number of } \\ \text { lesions }\end{array} \\ & & \end{array}$

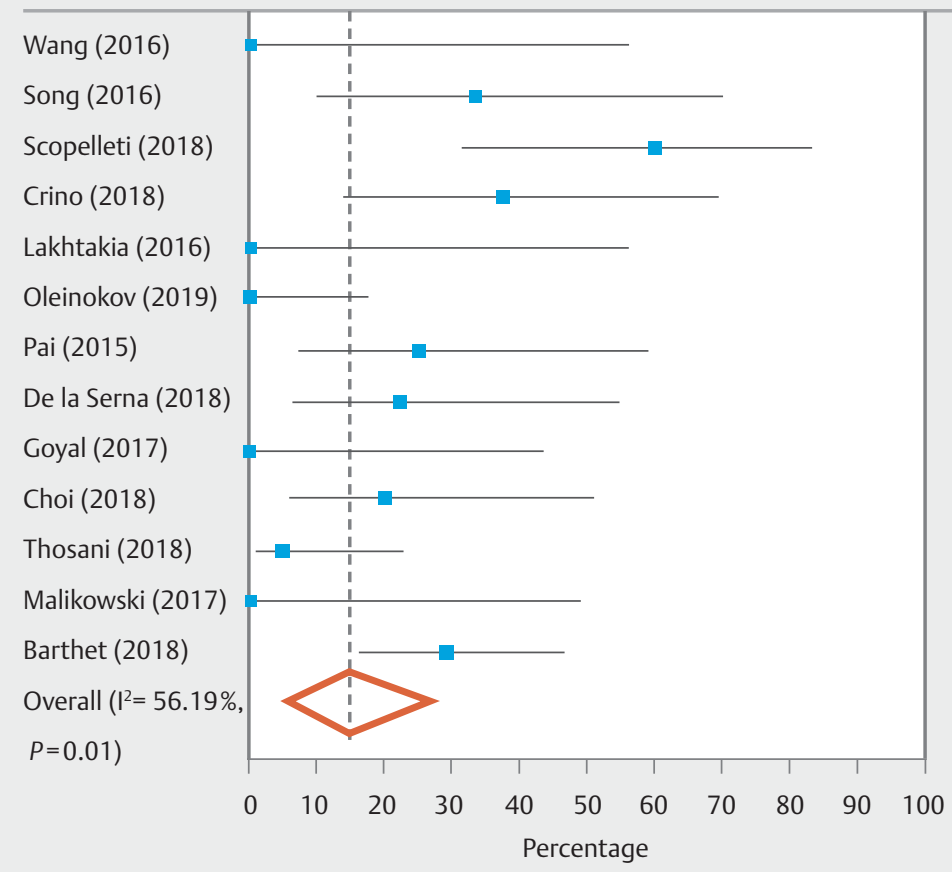

$\begin{array}{rrrr}0.00(0.00,56.15) & 4.48 & 0 & 3 \\ 33.33(9.68,70.00) & 6.62 & 2 & 6 \\ 60.00(31.27,83.18) & 8.40 & 6 & 10 \\ 37.50(13.68,69.43) & 7.62 & 3 & 8 \\ 0.00(0.00,56.15) & 4.48 & 0 & 3 \\ 0.00(0.00,17.59) & 10.36 & 0 & 18 \\ 25.00(7.15,59.07) & 7.62 & 2 & 8 \\ 22.22(6.32,54.74) & 8.03 & 2 & 9 \\ 0.00(0.00,43.45) & 6.01 & 0 & 5 \\ 20.00(5.67,50.98) & 8.40 & 2 & 10 \\ 4.67(0.85,22.67) & 10.83 & 1 & 21 \\ 0.00(0.00,48.99) & 5.30 & 0 & 4 \\ 29.03(16.10,46.59) & 11.86 & 9 & 31 \\ 14.67(4.77,27.46) & 100.00 & & \end{array}$

Fig. 4 Overall pooled total early adverse events.

- Table 4 Early and delayed adverse events in EUS-RFA.

\begin{tabular}{|c|c|c|c|c|c|c|c|c|}
\hline Author & $\begin{array}{l}\text { Total early } \\
\text { adverse } \\
\text { events }\end{array}$ & Pancreatitis & Bleeding & Perforation & Infection & $\begin{array}{l}\text { Abdominal } \\
\text { pain }\end{array}$ & Others & $\begin{array}{l}\text { Late adverse } \\
\text { events }\end{array}$ \\
\hline Oleinikov [11] & 0 & 0 & 0 & 0 & 0 & 0 & 0 & 2 \\
\hline Barthet [1] & 9 & 1 & 0 & 1 & 1 & 6 & 0 & 1 \\
\hline Scopelliti [3] & 6 & 0 & 0 & 0 & 0 & 2 & 4 & 0 \\
\hline Crino [9] & 3 & 0 & 0 & 0 & 0 & 3 & 0 & 0 \\
\hline De la Serna [8] & 2 & 0 & 0 & 0 & 0 & 2 & 0 & 0 \\
\hline Choi [7] & 2 & 1 & 0 & 0 & 0 & 1 & 0 & 0 \\
\hline Thosani [12] & 1 & 0 & 0 & 0 & 0 & 1 & 0 & 0 \\
\hline Goyal [13] & 0 & 0 & 0 & 0 & 0 & 0 & 0 & 0 \\
\hline Malikowski [14] & 0 & 0 & 0 & 0 & 0 & 0 & 0 & 0 \\
\hline Wang [16] & 0 & 0 & 0 & 0 & 0 & 0 & 0 & 0 \\
\hline Song [4] & 2 & 0 & 0 & 0 & 0 & 2 & 0 & 0 \\
\hline Lakhtakia [10] & 0 & 0 & 0 & 0 & 0 & 0 & 0 & 0 \\
\hline Pai [15] & 2 & 0 & 0 & 0 & 0 & 2 & 0 & - \\
\hline
\end{tabular}


mor size, necrosis of tumor as evidenced by hypo-enhanced areas noted on post-intervention CTs, and/or decrease in symptoms caused by functioning pancreatic tumors. Ten of 13 studies noted the clinical success rate $[1,3-11,15,16]$. Of these, six studies showed $100 \%$ pooled clinical success rates with the remaining four reporting clinical success rates ranging from $70 \%$ to $83 \%$. Many noted a sustained linear regression of the tumor with time when comparing CTs from 7 days post-procedure to CTs at 30 days post-procedure $[1,3,10,11]$.

No significant correlation was found between ablation time, radiofrequency strength, and sustained reduction in tumor burden. For example, Crino et al used $30 \mathrm{~W}$ to produce a $30 \%$ reduction in locally advanced PDAC, while Choi et al employed 20 to $50 \mathrm{~W}$ to see a $58.9 \%$ reduction in tumor size $[7,9]$. Multiple sessions of EUS-RFA were employed in all studies.

EUS-RFA can be used successfully to treat PNETs regulated to surveillance, or to ablate lesions in patients who refuse surveillance or decline surgery due to its invasiveness; individuals who are not surgical candidates because of comorbidities; and young patients with PNETs associated with MEN-1 in whom pancreas-sparing options are preferable [23]. In non-functional PNETs, Berthet et al noted $86 \%$ had diminished by at least $50 \%$ in size or completely by 12 months following ablation [1]. Functional PNETs exhibited a sustained attenuation of clinical symptoms such as hypoglycemia or diarrhea, rapid normalization of secreted hormone levels, and sustained significant decrease in size of the neoplasm. PNETs had a pooled clinical success rate ranging from $83 \%$ to $100 \%$ [10-13].

The secondary endpoint of this meta-analysis was to analyze AEs associated with EUS-RFA. The overall pooled incidence of AEs was $14.67 \%$ (95\% Cl [4.77-27.46], $\left.\mathrm{I}^{2}=56.19 \%\right)$. AEs were divided into early ( $<7$ days) and late ( $>7$ days). The most common early $A E$ was self-resolving abdominal pain $(9.82 \%(95 \% \mathrm{Cl}$ [3.34-18.24], $\left.\mathrm{I}^{2}=23.76 \%\right)$ ). There was one report of self-resolving pancreatitis. In that instance, Choi et al. recommended a 5 -mm margin from the pancreatic duct to avoid pancreatitis. Delayed AEs were reported in two studies [1, 3]. In all patients, no correlation was found between AEs and ablation time or energy settings.

Limitations of our meta-analysis include the fact that 10 of 13 studies were single-center studies with 10 or fewer patients $[3,4,7,9,10,13-16]$. Our meta-analysis also showed moderate to substantial heterogeneity. The studies pertaining to pancreatic neuroendocrine tumors all had short follow-up periods under 1 year $[7,8,10,11,14]$.

\section{Conclusion}

Overall, EUS-RFA has exhibited both high technical and clinical success with minimal AEs in addressing locally advanced unresectable PDAC and other pre-malignant pancreatic lesions where curative surgery is not an option. In the future, EUS-RFA may become a more widely used approach to treatment of a myriad of pancreatic lesions. Further long-term multicenter prospective studies are needed to correlate our findings.

\section{Competing interests}

The authors declare that they have no conflict of interest.

\section{References}

[1] Barthet M, Giovannini M, Lesavre N et al. Endoscopic ultrasoundguided radiofrequency ablation for pancreatic neuroendocrine tumors and pancreatic cystic neoplasms: a prospective multicenter study. Endoscopy 2019; 51: 836-842

[2] National Comprehensive Cancer Network. NCCN Clinical practice guidelines in oncology. Pancreatic adenocarcinoma.Version 2. 2016: http://wwwnccnorg/professionals/physician_gls/pdf/pancreaticpdf

[3] Scopelliti F, Pea A, Conigliaro R et al. Technique, safety, and feasibility of EUS-guided radiofrequency ablation in unresectable pancreatic cancer. Surg Endosc 2018; 32: 4022-4028

[4] Song TJ, Seo DW, Lakhtakia S et al. Initial experience of EUS-guided radiofrequency ablation of unresectable pancreatic cancer. Gastrointest Endosc 2016; 83: 440-443

[5] Moher D, Liberati A, Tetzlaff J et al. Preferred reporting items for systematic reviews and meta-analyses: the PRISMA statement. Annals Int Medic 2009; 151: 264-269

[6] Stang A. Critical evaluation of the Newcastle-Ottawa scale for the assessment of the quality of nonrandomized studies in meta-analyses. Eur J Epidemiol 2010; 25: 603-605

[7] Choi JH, Seo DW, Song TJ et al. Endoscopic ultrasound-guided radiofrequency ablation for management of benign solid pancreatic tumors. Endoscopy 2018; 50: 1099-1104

[8] De la Serna C, Cimavilla M, Madrigal B et al. Su1384 EUS-Guided radiofrequency ablation (EUS-RFA) for pancreatic neuroendocrine tumors and cystic mucinous neoplasms: a pilot study of safety, feasibility and efficacy. Gastrointest Endosc 2018; 87: AB332-AB333

[9] Crino SF, D'Onofrio M, Bernardoni L et al. EUS-guided Radiofrequency Ablation (EUS-RFA) of Solid Pancreatic Neoplasm Using an 18-gauge Needle Electrode: Feasibility, Safety, and Technical Success. J Gastrointestin Liver Dis 2018; 27: 67-72

[10] Lakhtakia S, Ramchandani M, Galasso D et al. EUS-guided radiofrequency ablation for management of pancreatic insulinoma by using a novel needle electrode (with videos). Gastrointest Endosc 2016; 83: 234-239

[11] Oleinikov K, Dancour A, Epshtein J et al. Endoscopic ultrasound-guided radiofrequency ablation: a new therapeutic approach for pancreatic neuroendocrine tumors. J Clin Endocrinol Metab 2019; 104: 2637-2647

[12] Thosani N, Sharma NR, Raijman I et al. 483 Safety and efficacy of endoscopic ultrasound guided radiofrequency ablation (EUS-RFA) in the treatment of pancreatic lesions: a multi-center experience. Gastrointest Endosc 2018; 87: AB84

[13] Goyal D, Cen P, Wray C] et al. 1111 Feasibility, Safety, and efficacy of endoscopic ultrasound (eus) guided radiofrequency ablation (RFA) of the pancreatic lesions: single center US experience. Gastrointest Endosc 2017; 85: AB144

[14] Malikowski T, Gleeson FC, Block M et al. Mo1268 Endoscopic ultrasound guided radiofrequency ablation (EUS-RFA): initial assessment of safety and efficacy. Gastrointest Endosc 2017; 85: AB484

[15] Pai M, Habib N, Senturk $\mathrm{H}$ et al. Endoscopic ultrasound guided radiofrequency ablation, for pancreatic cystic neoplasms and neuroendocrine tumors. World J Gastrointest Surg 2015; 7: 52-59

[16] Wang D, Jin Z, Lei W et al. Mo1524 endoscopic ultrasound guided radiofrequency ablation for the treatment of advanced pancreatic carcinoma. Gastrointest Endosc 2013; 77: AB414 
[17] Barret M, Leblanc S, Rouquette A et al. EUS-guided pancreatic radiofrequency ablation: preclinical comparison of two currently available devices in a pig model. Endosc Int Open 2019; 7: E138-E143

[18] DerSimonian R, Laird N. Meta-analysis in clinical trials. Control Clin Trials 1986; 7: 177-188

[19] Sutton AJ, Abrams KR, Jones DR et al. Methods for meta-analysis in medical research. Chichester: Wiley; 2000

[20] Higgins JPT, Thompson SG, Deeks J] et al. Measuring inconsistency in meta-analyses. BMJ 2003; 327: 557-560
[21] Kanwal F, White D. "Systematic Reviews and Meta-analyses" in clinical gastroenterology and hepatology. Clin Gastroenterol Hepatol 2012; 10: $1184-1186$

[22] Guyatt GH, Oxman AD, Kunz R et al. GRADE guidelines: 7. Rating the quality of evidence-inconsistency. J Clin Epidemiol 2011; 64: 12941302

[23] Larghi A, Rizzatti G, Rimbaş M et al. EUS-guided radiofrequency ablation as an alternative to surgery for pancreatic neuroendocrine neoplasms: Who should we treat? Endosco Ultrasound 2019; 8: 220-226 